\title{
ASTROPARTICLE PHYSICS TODAY
}

\author{
Franco Giovannelli*
}

\author{
INAF - Istituto di Astrofisica e Planetologia Spaziali, Area di Ricerca di Tor Vergata, Via del Fosso del \\ Cavaliere, 100 - I 00133 Roma, Italy \\ * corresponding author: franco.giovannelli@iaps.inaf.it
}

ABSTRACT. In this short review paper I will draw attention to the most important steps made in the past decade toward a better understanding of the physics governing our Universe. The results that I will discuss are drawn from the photonic astrophysics, particle astrophysics, and neutrino astrophysics, which constitute the main tools for exploring the Universe. The union of these three tools has given rise to a new field of physics known as Astroparticle Physics. Because of the limited length of this paper, I have selected only a few arguments that, in my opinion, have been crucial for the progress of Physics.

KEYWORDS: photonic astrophysics, particle astrophysics, neutrino astrophysics.

\section{INTRODUCTION}

Astroparticle Physics, a new field of physics, was formed about twenty years ago by joining the efforts of the community of High Energy Astrophysicists and the community of Particle Physicists. Over this relatively short period of time, Astroparticle Physics has developed strongly through the study of cosmic sources that emit photons, charged particles, and neutrinos. These sources are considered as Frontier Objects between astrophysics and particle physics. Results emerging from the study of cosmic sources via photonic astrophysics, particle astrophysics, and neutrino astrophysics have been stimulating the scientific community toward a unifying scheme for general comprehension of the physics governing our Universe.

The WMAP (Wilkinson Microwave Anisotropy Probe) Mission described by Bennett et al. (2003) determined that the universe is $13.7 \pm 0.2 \mathrm{Gyr}$ old. The combination of WMAP and 2dFGRS data constrains the energy density in stable neutrinos: $\Omega_{\nu} h^{2}<0.0072$. For three degenerate neutrino species, this limit implies that their mass is less than $0.23 \mathrm{eV}$ (95\% confidence limit). The best fit of the data favors a flat universe, from which it follows that the mean energy density in the universe is equal to the critical density (Spergel et al., 2003). This is equivalent to a mass density of $9.9 \times 10^{-30} \mathrm{~g} \mathrm{~cm}^{-3}$, which is equivalent to only 5.9 protons $\mathrm{m}^{-3}$. Of this total density, we now know the breakdown to be:

4.6\% baryons. This includes stars, heavy elements, and helium and free hydrogen.

$\mathbf{0 . 4 \%}$ neutrinos. Fast-moving neutrinos do not play a major role in the evolution of structure in the universe. They would have prevented the early clumping of gas in the universe, delaying the emergence of the first stars, in conflict with the WMAP data. However, with 5 years of data, WMAP is able to show evidence that a sea of cosmic neutrinos do exist in numbers that are expected from other lines of reasoning. This is the first time that such evidence has come from the cosmic microwave background.

23 \% Cold Dark Matter. Dark matter is likely to be composed of one or more species of sub-atomic particles that interact very weakly with ordinary matter. Several plausible candidates for dark matter exist. New particle accelerator experiments, and especially the Large Hadron Collider (LHC), are likely to provide new insights in the coming years.

$72 \%$ Dark Energy. The first observational hints of dark energy in the universe date back to the 1980s when astronomers were trying to understand how clusters of galaxies were formed. Their attempts to explain the observed distribution of galaxies were improved if dark energy was present, but the evidence was highly uncertain. In the 1990s, observations of supernovae were used to trace the expansion history of the universe, and it was a big surprise that the expansion appeared to be accelerated, rather than decelerated. In 2003, the first WMAP results came out, indicating that the universe was flat, and that dark matter made up only $\sim 23 \%$ of the density required to produce a flat universe. If $72 \%$ of the energy density in the universe is in the form of dark energy, which has a gravitationally repulsive effect, this is just the right amount to explain both the flatness of the universe and the observed accelerated expansion. Thus dark energy explains many cosmological observations all at the same time (NASA Official Web page, Edward J. Wollack, 2011),

However, we have no experimental proofs up till now of the existence of dark matter and dark energy. Thus we can affirm with reasonable certainty that the ocean composed of dark matter and dark energy is equivalent to the place outside the Roman World where the Ancient Romans placed lions (Hic sunt leones). We know rather well, but not yet completely, 
about only $5 \%$ of the content of the Universe!

Photonic astrophysics, particle astrophysics and neutrino astrophysics are the three tools that have made it possible to sound this $5 \%$ portion of the universe. The combined use of these three tools has given rise to a new field of physics, known as Astroparticle Physics.

All cosmic sources, both discrete and diffuse, are variable at different time scales in intensity and in spectral shape. In this sense, we can affirm that no one source is sufficiently stable to be considered as a standard candle. For this reason, multifrequency observations, possibly simultaneous, are mandatory for a proper comprehension of the behaviour of a target cosmic source.

A clear example of this assertion is the case of the Crab nebula which was considered very stable, like a standard candle, until the strong flares detected by the AGILE satellite in October 2007 and September 2010 in the $100 \mathrm{MeV}-10 \mathrm{GeV}$ energy range with an intensity three times greater than in quiescence (Tavani et al., 2011), and by the FERMI Gamma-ray space telescope in February 2009 and September 2010 at energies $>100 \mathrm{MeV}$, with intensity of four and six times greater than in quiescence, respectively (Abdo et al., 2011). The brevity of the flares implies that the $\gamma$-rays were emitted via synchrotron radiation from $10^{15} \mathrm{eV}$ electrons in a region smaller than $1.4 \times 10^{-2} \mathrm{pc}$. These are the highest energy particles that can be associated with a discrete astronomical source, and they pose challenges to particle acceleration theory.

In this paper I will discuss, on the basis of my knowledge and feelings, the most relevant results obtained in the recent past that have significantly improved our knowledge of the physics governing our universe. Deeper discussions about astroparticle physics can be found in the review papers by Giovannelli (2007, 2009, 2011). In their reviews, Giovannelli \& SabauGraziati (2010, 2012a), and De Angelis, Mansutti \& Persic (2008) have discussed papers about the multifrequency behaviour of high energy cosmic sources, and very high energy (VHE) $\gamma$-ray astrophysics.

\section{Astroparticle PHYSICS DEVELOPMENT}

High Energy Astrophysics is generally approached through the study of cosmic rays. The reason for this is historical in nature. Since the discovery of this extraterrestrial radiation by Victor Hess (1912), an enormous amount of scientific research has attempted to discover its nature, and as a result many separate research fields have been developed. Before particle accelerators came into operation, high energy cosmic rays were the laboratory tools for investigations of elementary particle production, and to date they remain the only source of particles with energies greater than $10^{12} \mathrm{eV}$. Research on the composition of this radiation led to the development of the study of the astrophysical environment using information in the charge, mass, and energy spectra; this field is known as Particle Astrophysics.

Now, the Large Hadron Collider (LHC), described by Straessner et al. (2011), is able to reach such Tev energies for $\mathrm{p}$-p interactions at $7 \mathrm{TeV}$ in searching for the Higgs Boson with the ATLAS Detector (Aad et al., 2012). No significant excess of events over the expected background is observed, and limits on the Higgs boson production cross section are derived for Higgs boson mass in the range $110 \mathrm{GeV}<m_{H}<$ $300 \mathrm{GeV}$. The observations exclude the presence of a standard model Higgs boson with mass $145 \mathrm{GeV}<$ $m_{H}<206 \mathrm{GeV}$ at a $95 \%$ confidence level.

Of great importance was the discovery of high energy photons near the top of the Earth's atmosphere. This discovery sparked the development of new astronomical fields such as X-ray and $\gamma$-ray astronomy. However, many of these high energy photons have their origin in interactions of the high energy charged particles with cosmic matter, light, or magnetic fields. In this fact, the fields of particle astrophysics and astronomical research have found a bond to join their efforts in trying to understand the high energy processes that occur in astrophysical systems.

\subsection{COSMIC RAYS}

The modern picture of cosmic rays is of a steady rain of particles moving at speeds close to the speed of light. The particles are primarily nuclei with atomic weights less than 56, as well as a few nuclei of heavier elements, some electrons and positrons, and a few $\gamma$-rays and neutrinos.

The energy spectrum extends over 12 orders of magnitude $\left(\sim 10^{8}-10^{20} \mathrm{eV}\right)$, and the particle flux decreases rapidly with increasing energy. Figure 1 (after Nagano \& Watson, 2000 and the references therein) shows the energy spectrum, starting from $10^{11} \mathrm{eV}$. The flux is multiplied by $E^{3}$ in order to keep the plot more compact, and to emphasize the variations in the spectrum.

A simple inspection of Fig. 1 shows a break in the spectrum around $10^{15}-10^{16} \mathrm{eV}$. This break is also called the knee. The knee was found at the end of the 1950 s, initially as the steepening in the EAS size spectrum (Kulikov \& Khristiansen, 1958). Since then, more than 60 years have passed but the origin of the $k n e e$ is still a challenge for cosmic ray physics. The steepening appears when the intensity of the showers falls to a value of $\sim 4-5 \times 10^{-11} \mathrm{~cm}^{-2} \mathrm{~s}^{-1} \mathrm{sr}^{-1}$. This intensity delivers one shower above the knee per area of $\sim 25 \mathrm{~m}^{2}$ in one hour. Owing to this low intensity the knee has not yet been studied by direct measurements in space, but it has been studied by indirect methods which use atmospheric cascades.

In summary, the cosmic ray spectrum for protons at $\mathrm{GeV}$ energies is close to $E^{-2.75}$, and for $\mathrm{He}$ and higher elements it is close to $E^{-2.65}$ below the knee at $\sim 5 \times 10^{15} \mathrm{eV}$, where the spectrum turns down to $\sim E^{-3.1}$, and flattens out again near $10^{18.5} \mathrm{eV}$, 


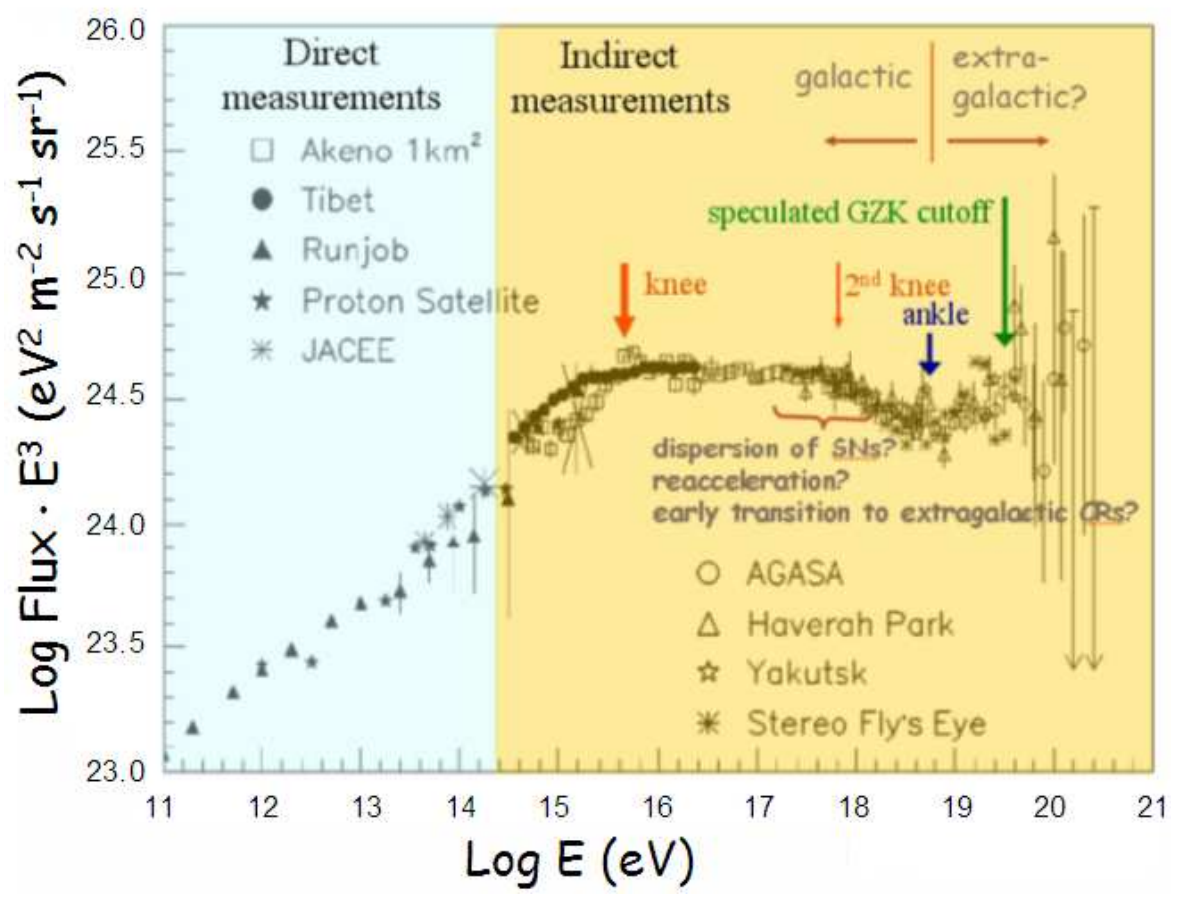

FiguRE 1. The all-particle spectrum of cosmic rays obtained by several experiments cited in the figure. Energies less than or greater than $4 \times 10^{14} \mathrm{eV}$ divide the two ranges in which direct and indirect measurements of the CR spectrum are possible. The knee, the ankle and the Greisen-Zatsepin-Kuzmin (GZK) cutoff, and a 2nd knee are clearly shown (after Nagano \& Watson, 2000).

called the ankle (e.g. Lawrence, Reid \& Watson, 1991; Nagano et al., 1992; Zatsepin, 1995).

The origin of the knee can be clarified by a detailed study of the primary spectrum, mass composition and arrival directions of cosmic rays. Reviews by Erlykin (1995), Müller (1993), and Hillas (1999) discussed these topics in detail. In particular, Erlykin (1995) provides a very clear discussion of the mass composition and the origin of cosmic rays. Indeed, their origin, independent of all the discussions and models, is focused around four main possible reasons, namely:

(1.) sources of cosmic rays;

(2.) acceleration mechanisms;

(3.) propagation through ISM;

(4.) interaction characteristics.

In most models based on one of these four items, the mass composition varies with the energy in various ways. Then Erlykin (1995) discussed the mass composition below $10^{5} \mathrm{GeV}$, near the knee $\left(10^{5}-10^{8} \mathrm{GeV}\right)$, and above $10^{8} \mathrm{GeV}$. He concluded that most of the results indicated that the genesis of cosmic rays is much more complicated than had been thought in the past. The most likely tendency for cosmic rays is to grow heavier with rising energies up to the knee region, and then to become lighter beyond the knee. Several reviews have been published about the chemical composition around the knee (Alessandro, 2001), about ultra high energy (UHE) cosmic rays (Blasi, 2001), and about the propagation and clustering of cosmic rays (Stanev, 2001).
A possible interpretation of the knee is that it represents the energy at which cosmic rays can escape more freely from the Galaxy or it may indicate a transition between two different acceleration mechanisms. In the first case one might expect an anisotropic effect in the distribution of the arrival directions above this energy if the cosmic rays were originated within the Galaxy.

Shibata (2008) discusses the status of the composition measurement of cosmic rays by direct observations and indirect observations which provides key information on the origin of cosmic rays. The results of the Tibet hybrid experiment measuring proton and the helium spectra at knee show the decreasing fractions of both the proton and helium component to the whole particle spectrum with increasing energy. This suggests that the knee is dominated by heavy nuclei.

A 2nd knee is present at about $10^{18} \mathrm{eV}$. Its origin is not yet completely clear: it could be due to dispersion of SNs, or due to reacceleration of particles or an early transition to extragalactic cosmic rays (e.g. Nagano \& Watson, 2000).

A summary on the status of the search for the origin of the highest energy cosmic rays has been published by Biermann (1999). He mentioned several competing proposals, such as supersymmetric particles, Gamma Ray Bursts also giving rise to energetic protons, interacting high energy neutrinos and cosmological defects, and then he discussed the possibility of the propagation of these particles, assuming that they are charged. The distribution of the arrival directions of the highest energy particles on the sky ought to reflect the source 
distribution and also the propagation history. He remarked that the present status can be summarized as inconclusive. However, he concluded as follows: If we can identify the origin of the events at the highest energies, beyond $5 \times 10^{19} \mathrm{eV}$, the Greisen-ZatsepinKuzmin cutoff owed to the microwave background, near to $10^{21} \mathrm{eV}$, and if we can establish the nature of their propagation through the universe to us, then we will obtain a tool to do physics at $\mathrm{EeV}$ energies.

The arrival directions of $\geq 60 \mathrm{EeV}$ ultra-high-energy cosmic rays (UHECRs) cluster along the supergalactic plane and correlate with active galactic nuclei (AGN) within $\sim 100 \mathrm{Mpc}$ (Abraham et al., 2007, 2008). The association of several events with the nearby radio galaxy Cen A supports the paradigm that UHECRs are powered by supermassive black-hole engines and are accelerated to ultra-high energies in the shocks formed by variable plasma winds in the inner jets of radio galaxies. The GZK horizon length of $75 \mathrm{EeV}$ UHECR protons is $\sim 100 \mathrm{Mpc}$, so that the Auger results are consistent with the assumed proton composition of UHECRs. In this scenario, the sources of UHECRs are FR II radio galaxies and FR I galaxies like Cen A with scattered radiation fields that enhance UHECR neutral beam production. Radio galaxies with jets pointed away from us can still be observed as UHECR sources due to the deflection of UHECRs by magnetic fields in the radio lobes of these galaxies. A broadband $\sim 1 \mathrm{MeV}-10 \mathrm{EeV}$ radiation component in the spectra of blazar AGN is formed by UHECR-induced cascade radiation in the extragalactic background light. This emission is too faint to be seen from Cen A, but could be detected from more luminous blazars (Dermer et al., 2009).

Recent evidence from the Pierre Auger Observatory suggests a transition, at $5 \mathrm{EeV}-10 \mathrm{EeV}$ in the composition of UHECRs, from protons to heavier nuclei such as iron (Abraham et al., 2010). Piran (2010) considered the implications of heavier composition on the sources of UHECRs. He concluded that with typical reasonable parameters of a few $\mathrm{nG}$ for the extra-galactic magnetic field (EGMF) intensity, and a coherence distance of an Mpc, the distance that nuclei UHECRs above GZK energy traverse before photodisintegrating is only a few Mpc. In spite of the significantly weaker limits on the luminosity, Cen A is the only currently active potential source of nuclei UHECRs within this distance. The large deflections erase the directional anisotropy expected from a single source. If indeed the composition of the above GZKUHECRs is iron, and if EGMF is not too small, then Cen $\mathrm{A}$ is the dominant source of the nuclei UHECRs observed above the GZK limit.

In summary, charged cosmic rays are influenced in their propagation through space by the magnetic fields in the Galaxy, and for the lowest energy particles they are also influenced in the solar system. The result is that the distribution of arrival directions as the radiation enters the Earth's atmosphere is nearly isotropic. It is not possible to identify the sources of the cosmic rays by detecting them. However, in the high energy interactions produced at the source, electrically neutral particles such as photons, neutrons, and neutrinos are also produced and their trajectories are not deviated being directed from their point of origin to the observer. Owing to their short lifetime neutrons cannot survive the path length to the Earth (decay length $\sim 9 \mathrm{pc}$ at $1 \mathrm{PeV}$ ) and neutrinos do not interact efficiently in the atmosphere.

It is in this context that Gamma Ray Astronomy has demonstrated itself to be a powerful tool. The observations made to date have detected $\gamma$-rays from many astronomical objects, e.g. neutron stars, interstellar clouds, the center of our Galaxy and the nuclei of active galaxies (AGNs). One might expect very important implications for high energy astrophysics from the observations at energies greater than $10^{11} \mathrm{eV}$ of extragalactic sources (e.g. Hillas \& Johnson, 1990). The fluxes of $\gamma$-rays at these energies are attenuated because of their interactions with the cosmic radio, microwave, infrared and optical radiation fields. Measurements of the flux attenuation can then provide important information on the distribution of these fields. For example, the threshold energy for pair production in reactions of photons with $2.7 \mathrm{~K}$ background radiation is reached at $10^{14} \mathrm{eV}$ and the absorption length is of the order of $\sim 7 \mathrm{kpc}$. For the infrared background the maximum absorption is reached at energies greater than $10^{12} \mathrm{eV}$.

The qualitative problem of the origin of cosmic rays has practically been solved, whilst the quantitative problem in determining the fraction of these rays coming from the different possible sources remains open.

\subsection{TeV SOURCES}

The most exciting results of the last decade have been obtained in the field of VHE astrophysics, from various experiments (e.g. CGRO/EGRET, Wipple, HEGRA, CANGAROO, Celeste, Stacee, Tibet, HESS, VERITAS, MILAGRO, MAGIC) that have detected many VHE cosmic sources.

The high energy sky — with the exception of the Crab nebula, Vela X, and 3C 273 - was empty until the mid 1990s. Updated to 19th April 2012, the VHE sky $(E>100 \mathrm{GeV})$ is populated by 107 cosmic sources: 46 out of 107 being extragalactic and 61 galactic (http: //www.mppmu.mpg.de/ rwagner/sources/ or http: //tevcat.uchicago.edu).

One of the most interesting results has been the determination of the Spectral Energy Distribution (SED) of the Crab nebula, thanks to many measurements obtained through various $\mathrm{HE}-\mathrm{VHE}$ experiments (Albert et al., 2008b).

Another exciting result has been the detection of the first variable galactic $\mathrm{TeV}$ source, namely the binary pulsar PSR B1259-63 (Aharonian et al., 2005). The authors found that radio silence occurs during the 
time in which the pulsar is occulted by the excretion disk of the Be star.

The many detected sources representing various galactic and extragalactic source populations are supernova remnants (SNRs), pulsar wind nebulae (PWNe), giant molecular clouds (GMCs), star formation regions (SFRs), compact binary systems (CBSs), and active galactic nuclei (AGNs). Paredes \& Persic (2010) reviewed the results from the MAGIC Cherenkov telescope for most of the former class of sources. Models of TeV AGNs have been discussed by Lenain (2010).

\subsection{Diffuse Extragalactic Background RADIATION}

After the Big Bang the Universe started to expand with rapid cooling. The cosmic radiation observed now is probably a melting of various components which had their origin in various stages of the evolution as the results of various processes. This is the Diffuse Extragalactic Background Radiation (DEBRA), which, if observed in various energy ranges, allows the study of many astrophysical, cosmological, and particle physics phenomena (Ressell \& Turner, 1990). DEBRA has witnessed the whole history of the Universe from the Big Bang to the present time.

This history is marked by three main experimental witnesses supporting the Big Bang theory (e.g. Giovannelli \& Sabau-Graziati, 2008): the light element abundances (Burles, Nollett \& Turner, 2001); the CMBR temperature at various redshifts as determined by Srianand, Petitjean \& Ledoux (2000), and the references therein; the $\mathrm{CMB}$ at $z=0$ as result of $\operatorname{COBE}\left(T_{\mathrm{CMBR}}(0)=2.726 \pm 0.010 \mathrm{~K}\right)$, which is well fitted by a black body spectrum (Mather et al., 1994). At $z \simeq 2.34$, the CMBR temperature is: $6.0 \mathrm{~K}<T_{\mathrm{CMBR}}(2.34)<14.0 \mathrm{~K}$. The prediction from the Hot Big Bang: $T_{\mathrm{CMBR}}=T_{\mathrm{CMBR}}(0) \times(1+z)$ gives $T_{\mathrm{CMBR}}(2.34)=9.1 \mathrm{~K}$, which is consistent with the measurement (Srianand, Petitjean \& Ledoux, 2000).

\subsection{Reionization of the Universe}

After the epoch of recombination (last scattering) between $\sim 3.8 \times 10^{5}$ and $\sim 2 \times 10^{8} \mathrm{yr}(z \simeq 1000$ $20)$, the universe experienced the so-called Dark Ages, where the dark matter halos collapsed and merged until the appearance of the first sources of light. This ended the Dark Ages. The ultraviolet light from the first sources of light also changed the physical state of the gas (hydrogen and helium) that fills the Universe, from a neutral state to a nearly fully ionized state. This was the Reionization Era where the population III stars formed and as feedback the first SNe and GRBs. This occurred between $\sim 2-5 \times 10^{8}$ yr $(z \simeq 20$ $10)$. Soon after population II stars started to form and the second wave of reionization probably occurred and stopped at $\sim 9 \times 10^{8}$ yr $(z \simeq 6)$ after the Big Bang, and then the evolution of galaxies started (e.g. Djorgovski, 2004, 2005). Quasars, the brightest and most distant objects known, offer a window on the reionization era, because neutral hydrogen gas absorbs their ultraviolet light.

Reionization drastically changes the environment for galaxy formation and evolution, and in a hierarchical clustering scenario the galaxies responsible for reionization may be the seeds for the most massive galaxies in the local Universe. Reionization is the last global phase transition in the Universe. The reionization era is thus a cosmological milestone, marking the appearance of the first stars, galaxies and quasars.

Recent results obtained by Ouchi et al. (2010) are an important contribution toward solving this problem. Indeed, from the the Ly $\alpha$ luminosity function (LF), clustering measurements and the Ly $\alpha$ line profiles based on the largest sample to date of $207 \mathrm{Ly} \alpha$ emitters at $z=6.6$ on the $1 \mathrm{deg}^{2}$ sky of Subaru/XMMNewton Deep Survey field, Ouchi et al. (2010) found that the combination of various reionization models and observational results about the LF, clustering, and line profile indicates that there would exist a small decrease of the intergalactic medium's (IGM's) Ly $\alpha$ transmission owing to reionization, but that the hydrogen IGM is not highly neutral at $z=6.6$. Their neutral-hydrogen fraction constraint implies that the major reionization process took place at $z>\sim 7$. The W. M. Keck $10 \mathrm{~m}$ telescope has shown quasar SDSS J1148+5251 at a redshift of $6.41\left(\sim 12.6 \times 10^{9} \mathrm{yr}\right.$ ago). This is currently the most distant known quasar (Djorkovski, 2004). This measurement does not contradict the result found for the epoch of reionization. However, the search of the epoch of reionization remains one of the most important open problems for understanding the formation of the first stars, galaxies and quasars.

\subsection{Clusters of Galaxies}

The problems of the production and transport of heavy elements seem to have been resolved. Indeed, thermally driven galactic winds, e.g. from M82, have shown that only active galaxies with an ongoing starburst can enrich the intracluster medium (ICM) with metals. The amounts of metals in the the ICM are at least as high as the sum of the metals in all galaxies of the cluster (e.g. Tozzi et al., 2003). Several clusters of galaxies (CGs) with strong radio emission have been associated with EGRET sources. This is an important step in clarifying the nature of many unknown EGRET sources (Colafrancesco, 2002). However, in the first 11 months of the Fermi LAT CG monitoring program, no $\gamma$-ray emission from any of the monitored CGs was detected (Ackermann et al., 2010b).

In spite of many important results coming from satellites of the last decade, the hierarchical distribution of dark matter, and also the role of the intergalactic magnetic fields in CGs, are still open. Multifrequency simultaneous measurements with higher sensitivity instruments, in particular those in the hard 
X-ray and radio energy regions and optical-near infrared (NIR) could solve problems such as these.

\subsection{Dark Energy And Dark Matter}

By using various methods to determine the mass of galaxies, a discrepancy has been found that suggests that $\sim 95 \%$ of the universe is in a form that cannot be seen. This form of unknown content of the universe is the sum of Dark Energy (DE) and Dark Matter (DM). Colafrancesco (2003) provides a deep discussion about the New Cosmology.

The discovery of the nature of dark energy may provide an invaluable clue for understanding the nature and the dynamics of our universe. However, there is $\sim 30 \%$ of the matter content of the universe which is dark and still requires a detailed explanation. Baryonic DM consisting of MACHOs (Massive Astrophysical Compact Halo Objects) can yield only some fraction of the total amount of Dark Matter required by CMB observations. WIMPs (Weakly Interacting Massive Particles) (non-baryonic DM) can yield the needed cosmological amount of DM and its large scale distribution provided that it is "cold" enough. Several options have been proposed so far, e.g.:

(1.) light neutrinos with mass in the range $m_{\nu} \sim 10$ $30 \mathrm{eV}$;

(2.) light exotic particles like axions with mass in the range $m_{\text {axion }} \sim 10^{-5}-10^{-2} \mathrm{eV}$;

(3.) or weakly interacting massive particles like neutralinos with mass in the range $M_{\chi} \sim 10-1000 \mathrm{GeV}$, this last option being favored at the present time (see e.g. Ellis 2002).

EROS and MACHO, two experiments based on the gravitational microlensing, have been developed. Two lines of sight have been probed intensively: the Large Magellanic Cloud (LMC) and the Small Magellanic Cloud (SMC), located $52 \mathrm{kpc}$ and $63 \mathrm{kpc}$, respectively, from the Sun (Palanque-Delabrouille, 2003).

With 6 years of data towards LMC, the MACHO experiment published a most probable halo fraction between $8 \%$ and $50 \%$ in the form of $0.2 M_{\odot}$ objects (Alcock et al., 2000). Most of this range is excluded by the EROS exclusion limit, and in particular the MACHO preferred value of $20 \%$ of the halo.

Among experiments for searching WIMP dark matter candidates, PAMELA is devoted to a search for dark matter annihilation, antihelium (primordial antimatter), new matter in the Universe (strangelets?), a study of cosmic-ray propagation (light nuclei and isotopes), the electron spectrum (local sources?), solar physics and solar modulation, and the terrestrial magnetosphere. A comparison of PAMELA expectations with many other experiments has been discussed by Morselli (2007). Bruno (2011) discusses some results from PAMELA.

The search for DM is one of the main open problems in present-day astroparticle physics.

\subsection{The Galactic Center}

The Galactic Center (GC) is one of the most interesting places for testing theories in which frontier physics plays a fundamental role. There is an excellent review by Mezger, Duschl \& Zylka (1996), which discusses the physical state of stars and interstellar matter in the Galactic Bulge $(R \sim 0.3-3 \mathrm{kpc}$ from the dynamic center of the Galaxy), in the Nuclear Bulge $(R<0.3 \mathrm{kpc})$ and in the Sgr A Radio and GMC Complex (the central $\sim 50 \mathrm{pc}$ of the Milky Way). This review also reports a list of review papers and conference proceedings related to the Galactic Center, with bibliographic details. In the review paper by Giovannelli \& Sabau-Graziati (2004, and the references therein), multifrequency GC behaviour is also discussed.

LaRosa et al. (2000) presented a wide-field, high dynamic range, high-resolution, long-wavelength $(\lambda=$ $90 \mathrm{~cm}$ ) VLA image of the Galactic center region. This is the most accurate image of the GC. It is highly obscured in optical and soft X-rays; it shows a central compact object - a black hole candidate - with $M \sim$ $3.6 \times 10^{6} M_{\odot}$ (Genzel et al., 2003a), which coincides with the compact radio source Sgr A* [R.A. 174541.3 (hh mm ss); Dec.: -29 0022 (dd mm ss)]. Sgr A* in X-rays/infrared is highly variable (Genzel et al., 2003b).

$\mathrm{GC}$ is also a good candidate for indirect dark matter observations. Moreover, the detected excess of $\mathrm{HE}$ $\gamma$-rays at GC would be produced by neutralino annihilation in the dark matter halo. This excess could be better measured by the FERMI observatory.

\subsection{GAMMA-RAY BURSTS}

A theoretical description of GRBs is still an open and strongly controversial issue. The most popular descriptions are the fireball (FB) model (Meszaros \& Rees, 1992; Piran, 1999), the cannon ball (CB) model (Dar \& De Rújula, 2004), the spinnin-precessing jet (SPJ) model (Fargion, 2003a,b; Fargion \& Grossi, 2006), and the fireshell (Izzo et al., 2010) model, which comes directly from the electromagnetic black hole (EMBH) model (e.g. Ruffini et al., 2003 and the references therein). However, each model competes against the others.

Important implications on the origin of the highest redshift GRBs come from the detection of GRB 080913 at $z=6.7$ (Greiner et al., 2009) and GRB 090423 at $z \simeq 8.2$ (Tanvir et al., 2009). This means that we are really approaching the possibility of detecting GRBs at the end of the Dark Era, where the first Pop III stars appeared. Izzo et al. (2010) successfully discuss a theoretical interpretation of the GRB 090423 within their fireshell model.

Wang \& Dai (2009) studied the high-redshift star formation rate (SFR) up to $z \simeq 8.3$ considering the Swift GRBs tracing the star formation history and the cosmic metallicity evolution in various background cosmological models including $\Lambda \mathrm{CDM}$, quintessence, 
and quintessence with a time-varying equation of state and brane-world models. $\Lambda \mathrm{CDM}$ is the preferred model, and it is compared with other results.

Although major progress has been made in recent years, GRBs theory needs further investigation in the light of experimental data coming from old and new satellites, often coordinated, e.g. BeppoSAX or BATSE/RXTE or ASM/RXTE or IPN or HETE or INTEGRAL or SWIFT or AGILE or FERMI or MAXI.

\subsection{Extragalactic Background Light}

Space is filled with diffuse extragalactic background light (EBL), which is the sum of the starlight emitted by galaxies throughout the history of the universe. High energy $\gamma$-rays traversing cosmological distances are expected to be absorbed through their interactions with EBL by: $\gamma_{\mathrm{VHE}}+\gamma_{\mathrm{EBL}} \longrightarrow \mathrm{e}^{+} \mathrm{e}^{-}$. Then the $\gamma$-ray flux $\Phi$ is suppressed while travelling from the emission point to the detection point, as $\Phi=\Phi_{0} e^{-\tau(E, z)}$, where $\tau(E, z)$ is the opacity. The $e$-fold reduction $[\tau(E, z)=$ 1] is the Gamma Ray Horizon (GRH) (e.g. Blanch \& Martinez, 2005; Martinez, 2007).

Direct measurement of EBL is difficult at optical to infrared wavelengths because of the strong foreground radiation originating in the solar system. However, the measurement of EBL is important for VHE gamma-ray astronomy, and also for astronomers modelling star formation and galaxy evolution. Second in intensity only to the cosmic microwave background (CMB), optical and infrared (IR) EBL contains the imprint of galaxy evolution since the Big Bang. This includes the light produced during the formation and reprocessing of stars. Current measurements of EBL are reported in the paper by Schroedter (2005, and references therein). Schroedter used the available VHE spectra from six blazars. Later, the redshift region over which the gamma reaction history (GRH) can be constrained by observations was extended up to $z=0.536$. The upper EBL limit has been obtained on the basis of $3 \mathrm{C}$ 279 data (Albert et al., 2008a). The universe is more transparent to VHE gamma rays than expected. Thus many more AGNs could be seen at these energies.

Indeed, Abdo et al. (2009a) observed a number of TeV-selected AGNs during the first 5.5 months of observations with the Large Area Telescope (LAT) on board the Fermi Gamma-ray Space Telescope. Redshift-dependent evolution is detected in the spectra of objects detected at $\mathrm{GeV}$ and $\mathrm{TeV}$ energies. The most reasonable explanation for this is absorption on EBL. As such, this would be the first modelindependent evidence for absorption of $\gamma$-rays on EBL. By using a sample of $\gamma$-ray blazars with redshift up to $z \simeq 3$, and GRBs with redshift up to $z \simeq 4.3$, measured by Fermi/LAT, Abdo et al. (2010b) placed upper limits on the $\gamma$-ray opacity of the universe at various energies and redshifts and compared this with predictions from well-known EBL models. They found that EBL intensity in the optical-ultraviolet wavelengths as great as predicted by the "baseline" model of Stecker, Malkan \& Scully (2006) can be ruled out with high confidence.

\subsection{Relativistic Jets}

Relativistic jets have been found in numerous galactic and extragalactic cosmic sources in various energy bands. The emitted spectra of jets from cosmic sources of different nature are strongly dependent on the angle formed by the beam axis and the line of sight, and obviously by the Lorentz factor of the particles (e.g. Bednarek et al., 1990 and the references therein; Beall, Guillory \& Rose, 1999, 2009; Beall, 2002, 2003, 2008, 2009; Beall et al., 2006, 2007). Observations of jet sources at various frequencies can therefore provide new inputs for the comprehension of these extremely efficient carriers of energy, as for cosmological GRBs. The discovered analogy among $\mu-$ QSOs, QSOs, and GRBs is fundamental for studying the common physics governing these different classes of objects via $\mu$-QSOs, which are galactic, and then apparently brighter and with all processes occurring in time scales accessible by our experiments (e.g. Chaty, 1998). Chaty (2007) noted the importance of multifrequency observations of jet sources by means of measurements of GRS $1915+105$.

Dermer et al. (2009) suggest that UHECRs could come from black hole jets of radio galaxies. Spectral signatures associated with UHECR hadron acceleration in studies of radio galaxies and blazars with the FERMI observatory and ground-based $\gamma$-ray observatories can provide evidence for cosmic-ray particle acceleration in black hole plasma jets. Also in this case, $\gamma$-ray multifrequency observations $(\mathrm{MeV}-\mathrm{GeV}-$ $\mathrm{TeV}$ ) together with observations of $\mathrm{PeV}$ neutrinos could confirm whether black-hole jets in radio galaxies accelerate UHECRs.

Despite their frequent outburst activity, microquasars have never been unambiguously detected emitting high-energy gamma rays. Fermi/LAT has detected a variable high-energy source coinciding with the position of the X-ray binary and microquasar Cygnus X-3. Its identification with Cygnus X-3 is secured by the detection of its orbital period in gamma rays, as well as the correlation of the LAT flux with radio emission from the relativistic jets of Cygnus X-3. The $\gamma$-ray emission probably originates from within the binary system (Abdo et al., 2009b). The microquasar LS 5039 has also been unambiguously detected by Fermi/LAT its emission being modulated with a period of 3.9 days. Analyzing the spectrum, variable with the orbital phase, and having a cutoff, Abdo et al. (2009c) conclude that the $\gamma$-ray emission of LS 5039 is magnetospheric in origin, like that of the pulsars detected by Fermi. This experimental evidence of emission in the $\mathrm{GeV}$ region of microquasars opens an interesting window on the formation of relativistic jets. 


\subsection{Cataclysmic variables}

The detection of CVs by the INTEGRAL observatory (Barlow et al., 2006) has recently renewed the interest of high energy astrophysicists in these systems, and has lead to renewed involvement of the low energy astrophysical community. The detection of CVs having orbital periods inside the so-called Period Gap between 2 and 3 hours, which separates polars, experiencing gravitational radiation, from intermediate polars, experiencing magnetic braking, renders attractive the idea of physical continuity between the two classes. Further investigations are necessary in order to solve this important problem.

For a recent review on CVs see the paper by Giovannelli \& Sabau-Graziati (2012b).

\subsection{High Mass X-RAY Binaries}

For general reviews, see e.g. Giovannelli \& SabauGraziati $(2001,2004)$ and van den Heuvel (2009) and references therein.

HMXBs are young systems, with age $\leq 10^{7} \mathrm{yr}$, mainly located in the galactic plane (e.g. van Paradijs, 1998). A compact object, the secondary star, mostly a magnetized neutron star (X-ray pulsar) is orbiting around an early type star $(\mathrm{O}, \mathrm{B}, \mathrm{Be})$, the primary, with $M \geq 10 M_{\odot}$. The optical luminosity of the system is dominated by the early type star.

Such systems are the best laboratory for the study of accreting processes thanks to their relatively high luminosity in a large part of the electromagnetic spectrum. Because of the strong interactions between optical companion and collapsed object, the low and high energy processes are strictly related. In X-ray/Be binaries the mass loss processes are due to the rapid rotation of the Be star, the stellar wind and, sporadically, due to the expulsion of a casual quantity of matter essentially triggered by gravitational effects close to the periastron passage of the neutron star. The long orbital period ( $>10$ days) and the large eccentricity of the orbit $(>0.2)$ together with transient hard X-ray behavior are the main characteristics of these systems. Among the whole sample of galactic systems containing 114 X-ray pulsars (Johnstone, 2005), only a few have been extensively studied. Among these, system A $0535+26 / \mathrm{HDE} 245770$ is the best known, thanks to concomitant favorable causes which have rendered possible thirty seven years of coordinated multifrequency observations, most of them discussed e.g. by Giovannelli \& Sabau-Graziati (1992, 2008), Burger et al. (1996).

Accretion powered X-ray pulsars usually capture material from the optical companion via the stellar wind, since this primary star generally does not fill its Roche lobe. However, in some specific conditions (e.g. passage at the periastron of the neutron star) and in particular systems (e.g. A $0535+26 / \mathrm{HDE} 245770$ ), a temporary accretion disk can form around the neutron star behind the shock front of the stellar wind. This enhances the efficiency of the process of mass transfer from the primary star onto the secondary collapsed star, as discussed by Giovannelli \& Ziolkowski (1990) and by Giovannelli et al. (2007) in the case of A $0535+26$.

Giovannelli \& Sabau-Graziati (2011) discussed the history of the discovery of optical indicators of high energy emission in the prototype system A0535+26/HDE 245770 E Flavia' star, updated to the March-April 2010 event when a strong optical activity occurred roughly 8 days before the X-ray outburst (Caballero et al., 2010) that was predicted by Giovannelli, Gualandi \& Sabau-Graziati (2010). This optical indicator of an X-ray outburst, together with the whole history of the A0535+26 system, led to the conclusion that the periastron passage of the neutron star is scanned every $110.856 \pm 0.002$ days (optical orbital period) (Bartolini et al., 1983), and the X-ray outbursts are triggered starting from that moment and occur roughly after 8 days - the transit time of the material expelled from the primary for reaching the secondary.

However, it is still an important open problem how $\mathrm{X}$-ray outbursts are triggered in X-ray pulsars. This issue has given rise to controversy among astrophysicists.

Important news has also been coming also from $\mathrm{GeV}$ observations of HMXBs. Indeed, Abdo et al. (2009e) have presented the first results from the observations of LSI $+61^{\circ} 303$ using Fermi/LAT data obtained between 2008 August and 2009 March. Their results indicate variability that is consistent with the binary period, with the emission being modulated at 26.6 days. This constitutes the first detection of orbital periodicity in high-energy $\gamma$-rays $(20 \mathrm{MeV}-100 \mathrm{GeV})$. The light curve is characterized by a broad peak after the periastron, as well as a smaller peak just before the apastron. The spectrum is best represented by a power law with an exponential cutoff, yielding an overall flux above $100 \mathrm{MeV}$ of $\sim 0.82 \times 10^{-6} \mathrm{ph} \mathrm{cm}^{-2} \mathrm{~s}^{-1}$, with a cutoff at $\sim 6.3 \mathrm{GeV}$ and photon index $\gamma \simeq 2.21$. There is no significant spectral change with orbital phase. The phase of maximum emission, close to the periastron, hints at inverse Compton scattering as the main radiation mechanism. However, previous very high-energy gamma ray $(>100 \mathrm{GeV}$ ) observations by MAGIC and VERITAS show peak emission close to the apastron. This and the energy cutoff seen with Fermi suggest that the link between HE and VHE gamma rays is nontrivial. This is an open problem to be solved in future.

\subsection{Obscured Sources and Supergiant FAST X-RAY TRANSIENTS}

There are relevant INTEGRAL results about a new population of obscured sources and Supergiant Fast X-ray Transients (SFXTs) (Chaty \& Filliatre, 2005; Chaty, 2007; Rahoui et al., 2008; Chaty, 2008). The importance of the discovery of this new population is based on the constraints on the formation and 
evolution of HMXBs: does the dominant population of short-living systems - born with two very massive components - occur in a rich star-forming region? What will happen when the supergiant star dies? Are primary progenitors of NS/NS or NS/BH mergers good candidates for gravitational waves emitters? Can we find a link with short/hard $\gamma$-ray bursts?

\subsection{Ultra Compact Double DEGENERATED BINARIES}

Ultra-compact double-degenerated binaries (UCD) consist of two compact stars, which can be black holes, neutron stars or white dwarfs. In the case of two white dwarfs revolving around each other with an orbital period $P_{\text {orb }}<20 \mathrm{~min}$, the separation of the two components for a UCD with $P_{\text {orb }} \simeq 10 \mathrm{~min}$ or shorter is smaller than the diameter of Jupiter.

These UCDs are evolutionary remnants of low-mass binaries, and they are numerous in the Milky Way. The discovery of UCD hints interestingly at possible gravitational wave detection with the LISA observatory.

\subsection{Magnetars}

The discovery of magnetars (Anomalous X-ray Pulsars - AXPs - and Soft Gamma-ray Repeaters SGRs) is another very exciting result from recent years (Mereghetti \& Stella, 1995; van Paradijs, Taam \& van den Heuvel, 1995; and e.g. a review by Giovannelli \& Sabau-Graziati, 2004 and the references therein). Indeed, with a magnetic field intensity of order $10^{14}-10^{15} \mathrm{G}$ the question naturally arises: what kind of SN produces these AXPs and SGRs? Are the collapsed objects in AXPs and SGRs really neutron stars? (e.g. Hurley, 2008). With such high magnetic field intensity, an almost 'obvious' consequence can be derived: the corresponding dimension of the source must be $\sim 10 \mathrm{~m}$ (Giovannelli \& Sabau-Graziati, 2006). This could be the dimension of the acceleration zone in supercompact stars. Could they be quark stars?

Ghosh (2009) discussed some of the recent developments in the quark star physics along with the consequences of possible hadron-to-quark phase transition in a high density scenario of neutron stars, and their implications for Astroparticle Physics.

Important consequences could be derived by the experimentally demonstrated continuity among rotationpowered pulsars, magnetars, and millisecond pulsars, (Kuiper, 2007). However, the physical reason for this continuity remains unclear.

\section{Cross Sections of Nuclear REACTIONS IN StaRs}

Knowledge of the cross-sections of nuclear reactions occurring in the stars appears to be one of the most crucial points of all astroparticle physics. Direct measurements of the cross sections of the ${ }^{3} \mathrm{He}\left({ }^{4} \mathrm{He}, \gamma\right){ }^{7} \mathrm{Be}$ and ${ }^{7} \mathrm{Be}(\mathrm{p}, \gamma)^{8} \mathrm{Be}$ reactions of the $\mathrm{p}-\mathrm{p}$ chain and ${ }^{14} \mathrm{~N}(\mathrm{p}, \gamma){ }^{15} \mathrm{O}$ reaction of the CNO-cycle will allow a substantial improvement in our knowledge about stellar evolution.

The LUNA collaboration has already measured with good accuracy the key reactions $\mathrm{D}(\mathrm{p}, \gamma){ }^{3} \mathrm{He},{ }^{3} \mathrm{He}(\mathrm{D}$, p) ${ }^{4} \mathrm{He}$ and ${ }^{3} \mathrm{He}\left({ }^{4} \mathrm{He}, \gamma\right){ }^{7} \mathrm{Be}$. These measurements substantially reduces the theoretical uncertainty of $\mathrm{D}$, ${ }^{3} \mathrm{He},{ }^{7} \mathrm{Li}$ abundances. The $\mathrm{D}\left({ }^{4} \mathrm{He}, \gamma\right){ }^{6} \mathrm{Li}$ cross section, which is the key reaction for determining the primordial abundance of ${ }^{6} \mathrm{Li}$, will be measured in the near future (Gustavino, 2007, 2009 and 2011).

\section{Neutrino Astronomy}

For a short discussion about neutrino astronomy, see e.g. the paper by Giovannelli (2007 and the references therein), as well as all the papers of the Neutrino Astronomy Session, which were published in the proceedings of the Vulcano Workshops 2006, 2008, and 2010 (Giovannelli \& Mannocchi, 2007, 2009, 2011).

However, it should be noted that several papers have appeared about:

(1.) the sources of HE neutrinos (Aharonian, 2007) and diffuse neutrinos in the Galaxy (Evoli, Grasso \& Maccione, 2007);

(2.) Potential neutrino signals from galactic $\gamma$-ray sources (Kappes et al., 2007);

(3.) galactic cosmic-ray pevatrons with multi-TeV $\gamma$ rays and neutrinos (Gabici \& Aharonian, 2007);

(4.) results achieved with AMANDA: 32 galactic and extragalactic sources have been detected (Xu \& ICECube Collaboration, 2008); diffuse neutrino flux from the inner Galaxy (Taylor et al., 2008); discussions about VHE neutrino astronomic experiments (Cao, 2008). Important news and references can be found in the proceedings of the Les Rencontres de Physique de la Vallée d'Aoste (Greco, 2009, 2010).

News about neutrino oscillations has been reported by Mezzetto (2011). Angle $\Theta_{13}$ differs from zero: $\sin ^{2} \Theta_{13}=0.013$. This result opens the door to CP violation searches in the neutrino sector, with profound implications for our understanding of matterantimatter asymmetry in the universe.

\section{CONClusions AND REFLECTIONS}

I will conclude this brief and incomplete review with some comments about the topics discussed here.

(1.) Many ground-based and space-based experiments have been exploring the whole energy range of the $\mathrm{CR}$ spectrum from $\sim 1 \mathrm{GeV}$ to $\sim 10^{12} \mathrm{GeV}$, and many experiments are programmed for the near future. Significant improvements have been obtained in the definition of the CR spectrum for protons, electrons, positrons, antiprotons and all ions. Better results are expected in the near future. Particular interest is devoted to knowledge about extreme high energy CRs. 
(2.) Many experiments have been exploring cosmic sources along the whole electromagnetic spectrum, and new space-based and ground-based experiments are developing a tendency to explore processes at higher and higher energies, which are directly linking photonic astrophysics with particle astrophysics.

(3.) Particular attention is needed at the highest energies, where the cosmic ray spectrum extends to $10^{20} \mathrm{eV}$ (see Figure 1). However, the origins of these spectacularly high energy particles remains obscure. Particle energies of this magnitude imply that there is a range of elementary particle physics phenomena present near their acceleration sites which is beyond the ability of present day particle accelerators to explore. VHE $\gamma$-ray astronomy may catch a glimpse of these phenomena.

It is becoming increasingly clear that the energy régime covered VHE $\gamma$-ray astronomy will be able to address a number of significant scientific questions, which include:

(1.) What parameters determine the cut-off energy for pulsed $\gamma$-rays from pulsars?

(2.) What is the role of shell-type supernovae in the production of cosmic rays?

(3.) At what energies do AGN blazar spectra cut off?

(4.) Are gamma blazar spectral cut-offs intrinsic to the source or due to intergalactic absorption?

(5.) Is the dominant particle species in AGN jets leptonic or hadronic?

(6.) Can intergalactic absorption of the VHE emission of AGNs be a tool for calibrating the epoch of galaxy formation, the Hubble parameter, and the distance to $\gamma$-ray bursts?

(7.) Are there sources of $\gamma$-rays which are 'loud' at VHEs, but 'quiet' at other wavelengths?

The importance of Multifrequency Astrophysics and Multienergy Particle Astrophysics seems evident. There are many problems in performing simultaneous Multi-frequency, Multi-energy Multi-site, Multiinstrument, Multi-platform measurements, due to:

(1.) objective technological difficulties;

(2.) sharing common scientific objectives;

(3.) problems of scheduling and budgets;

(4.) political management of science.

In spite of the many ground-based and space-based experiments that provide an impressive quantity of excellent data in various energy regions, many open problems still exist. I believe that only a drastic change in the philosophy of the experiments will lead to faster solution of most of the problems that remain open. For example, in the case of space-based experiments, small satellites — dedicated to specific missions and problems, and able to schedule very long-term observations - must be supported, because they can be prepared relatively rapidly, are easier to manage and are less expensive than medium-size and large satellites.

I strongly believe that in the coming decades passive-physics experiments, space-based and groundbased and maybe also lunar-based, will be the most suitable probes for sounding the physics of the Universe. Active physics experiments have probably already reached the maximum dimensions compatible with a reasonable cost/benefit ratio, with the obvious exception of neutrino-astronomy experiments.

\section{ACKNOWLEDGEMENTS}

I wish to thank the LOC of the Karlovy Vary 9th INTEGRAL/BART Workshop for logistic support. This research has made use of NASA's Astrophysics Data System.

\section{REFERENCES}

[1] Aad, G. et al. (ATLAS Collaboration): 2012, Phys. Rev. Letter 108, 1802.

[2] Abdo, A.A. et al.: 2009a, ApJ 707, 1310.

[3] Abdo, A.A. et al.: 2009b, Sci. 326, 1512.

[4] Abdo, A.A. et al.: 2009c, ApJ 706, L56.

[5] Abdo, A.A. et al.: 2009e, ApJ 701L, 123.

[6] Abdo, A.A. et al.: 2010b, ApJ 723, 1082.

[7] Abdo, A.A.: 2011, Science 331, 739.

[8] Abraham, J. et al. (The Pierre Auger Collaboration): 2007, Science 318, 938.

[9] Abraham, J. et al. (The Pierre Auger Collaboration): 2008, Astropart. Phys. 29, 188.

[10] J. Abraham, J. et al.: 2010, Phys. Rev. Letter 104, 091101.

[11] Ackermann, M. et al.: 2010b, ApJ 717, L71.

[12] Aharonian, F.A.: 2007, Sci. 315, 70.

[13] Aharonian, F. et al.: 2005, A\&A 442, 1.

[14] Albert, J. et al. (MAGIC Collaboration): 2008a, Sci. 320,1752 .

[15] Albert, J. et al.: 2008b, ApJ 674, 1037.

[16] Alcock, C. et al.: 2000, ApJ 542, 281.

[17] Alessandro, B.: 2001, in Frontier Objects in Astrophysics and Particle Physics, F. Giovannelli \& G. Mannocchi (eds.), Italian Physical Society, Editrice Compositori, Bologna, Italy 73, 363.

[18] Barlow, E.J. et al.: 2006, MNRAS 372, 224.

[19] Bartolini, C., Bianco, G., Guarnieri, A., Piccioni, A., Giovannelli, F.: 1983, Hvar Obs. Bull. 7(1), 159.

[20] Beall, J.H.: 2002, in Multifrequency Behaviour of High Energy Cosmic Sources, F. Giovannelli \& L. Sabau-Graziati (eds.), Mem. SAIt 73, 379.

[21] Beall, J.H.: 2003, ChJA\&AS 3, 373.

[22] Beall, J.H.: 2008, ChJA\&AS 8, 311.

[23] Beall, J.H.: 2009, in Frontier Objects in Astrophysics and Particle Physics, F. Giovannelli \& G. Mannocchi, (eds.), Italian Physical Society, Ed. Compositori, Bologna, Italy, 98, 283. 
[24] Beall, J.H., Guillory, J., Rose, D.V.: 1999, in Multifrequency Behaviour of High Energy Cosmic Sources, F. Giovannelli \& L. Sabau-Graziati (eds.), Mem. SAIt 70, 1235.

[25] Beall, J.H. et al.: 2006, ChJA\&AS1 6, 283.

[26] Beall, J.H. et al.: 2007, in Frontier Objects in Astrophysics and Particle Physics, F. Giovannelli \& G. Mannocchi, (eds.), Italian Physical Society, Ed. Compositori, Bologna, Italy, 93, 315.

[27] Beall, J.H., Guillory, J., Rose, D.V.: 2009, in Frontier Objects in Astrophysics and Particle Physics, F. Giovannelli \& G. Mannocchi, (eds.), Italian Physical Society, Ed. Compositori, Bologna, Italy, 98, 301.

[28] Bednarek, W., Giovannelli, F., Karakula, S., Tkaczyk, W.: 1990, A\&A 236, 268

[29] Bennett, C.L. et al.: 2003, ApJ 583, 1.

[30] Biermann, P. L.: 1999, Astrophys. and Space Sci. 264,423

[31] Blanch, O., Martinez, M.: 2005, Astrop. Phys. 23, 588.

[32] Blasi, P.: 2001, Astrop. Phys. 15, 223.

[33] Bruno, A.: 2011, in Frontier Objects in Astrophysics and Particle Physics, F. Giovannelli \& G. Mannocchi (eds.), Italian Physical Society, Ed. Compositori, Bologna, Italy, 103, 139.

[34] Burger, M. et al.: 1996, in Multifrequency Behaviour of High Energy Cosmic Sources, F. Giovannelli \& L. Sabau-Graziati (eds.), Mem. SAIt 67, 365.

[35] Burles, S., Nollet, K.M., Turner, M.S.: 2001, ApJL 552 , L1.

[36] Caballero, I. et al.: 2010, ATEL No. 2541.

[37] Cao, Z.: 2008, Nucl. Phys. B (Proc. Suppl.) 175-176, 377 .

[38] Chaty, S.: 1998, Ph.D. thesis, University Paris XI.

[39] Chaty, S., 2007, in Frontier Objects in Astrophysics and Particle Physics, F. Giovannelli \& G. Mannocchi (eds.), Italian Physical Society, Ed. Compositori, Bologna, Italy, 93, 329.

[40] Chaty, S.: 2008, ChJA\&AS 8, 197.

[41] Chaty, S., Filliarte, P.: 2005, ChJA\&AS 5, 104.

[42] Colafrancesco, S.: 2002, A\&A 396, 31.

[43] Colafrancesco, S.: 2003, in Frontier Objects in Astrophysics and Particle Physics, F. Giovannelli \& G. Mannocchi (eds.), Italian Physical Society, Ed. Compositori, Bologna, Italy, 85, 141.

[44] Dar, A., De Rújula, A.: 2004, Phys. Rep. 405, 203.

[45] De Angelis, A., Mansutti, O., Persic, M.: 2008, Il N. Cim. 31 N. 4, 187.

[46] Dermer,C.D., Razzaque, S., Finke, J.D., Atoyan, A.: 2009, New J. of Phys. 11, 1.

[47] Djorgovski, S.G.: 2004, Nature 427, 790.

[48] Djorgovski, S.G.: 2005, in The Tenth Marcel Grossmann Meeting, M. Novello, S. Perez Bergliaffa \& R. Ruffini (eds.), World Scientific Publishing Co., p. 422.

[49] Ellis, J.: 2002, astro-ph 4059 (arXiv:hep-ex/0210052).

[50] Erlykin, A.D.: 1995, in Frontier Objects in Astrophysics and Particle Physics, F. Giovannelli \& G. Mannocchi (eds.), Italian Physical Society, Editrice Compositori, Bologna, Italy 47, 483.
[51] Evoli, C., Grasso, D., Maccione, L.: 2007, astro-ph 0701856

[52] Fargion, D.: 2003a, in Frontier Objects in Astrophysics and Particle Physics, F. Giovannelli \& G. Mannocchi (eds.), Italian Physical Society, Ed. Compositori, Bologna, Italy 85, 267.

[53] Fargion, D.: 2003b, ChJA\&AS 3, 472

[54] Fargion, D., Grossi, M.: 2006, ChJA\&AS1 6, 342.

[55] Gabici, S., Aharonian, F.A.: 2007, ApJL 665, L131.

[56] Genzel, R. et al.: 2003a, ApJ 594, 812.

[57] Genzel, R. et al.: 2003b, Nature 425, 934.

[58] Ghosh, S.K.: 2009, in Frontier Objects in Astrophysics and Particle Physics, F. Giovannelli \& G. Mannocchi, (eds.), Italian Physical Society, Ed. Compositori, Bologna, Italy, 98, 243.

[59] Giovannelli, F.: 2007, in Frontier Objects in Astrophysics and Particle Physics, F. Giovannelli \& G. Mannocchi (eds.), Italian Physical Society, Ed. Compositori, Bologna, Italy, 93, 3.

[60] Giovannelli, F.: 2009, in Frontier Objects in Astrophysics and Particle Physics, F. Giovannelli \& G. Mannocchi (eds.), Italian Physical Society, Ed. Compositori, Bologna, Italy, 98, 3.

[61] Giovannelli, F.: 2011, in Frontier Objects in Astrophysics and Particle Physics, F. Giovannelli \& G. Mannocchi (eds.), Italian Physical Society, Ed. Compositori, Bologna, Italy, 103, 3.

[62] Giovannelli, F., Ziółkowski, J.: 1990, AcA 40, 95.

[63] Giovannelli, F., Sabau-Graziati, L.: 1992, Space Sci. Rev. 59, 1.

[64] Giovannelli, F., Sabau-Graziati, L.: 2001, Ap\&SS $276,67$.

[65] Giovannelli, F., Sabau-Graziati, L.: 2004, Space Sci. Rev. 112, 1.

[66] Giovannelli, F., Sabau-Graziati, L.: 2006, ChJA\&AS1 6,1 .

[67] Giovannelli, F., Bernabei, S., Rossi, C., Sabau-Graziati, L.: 2007, A\&A 475, 651.

[68] Giovannelli, F., Mannocchi, G. (eds.): 2007, 2009, 2011, Proc. Vulcano Workshops on Frontier Objects in Astrophysics and Particle Physics, Italian Physical Society, Ed. Compositori, Bologna, Italy, Vol. 93, 98, 103.

[69] Giovannelli, F., Sabau-Graziati, L.: 2008, ChJA\&AS 8,1 .

[70] Giovannelli, F., Gualandi, R., Sabau-Graziati, L.: 2010, ATEL No. 2497.

[71] Giovannelli, F., Sabau-Graziati, L.: 2010, in Multifrequency Behaviour of High Energy Cosmic Sources, F. Giovannelli \& L. Sabau-Graziati (eds.), Mem. SAIt 81 N. 1, 18 .

[72] Giovannelli, F., Sabau-Graziati, L.: 2011, Acta Polyt. 51 N. 2, 21.

[73] Giovannelli, F., Sabau-Graziati, L.: 2012a, in Multifrequency Behaviour of High Energy Cosmic Sources, F. Giovannelli \& L. Sabau-Graziati (eds.), Mem. SAIt 83 N. 1, 17 . 
[74] Giovannelli, F., Sabau-Graziati, L.: 2012b, in The Golden Age of Cataclysmic Variables and Related Objects, F. Giovannelli \& L. Sabau-Graziati (eds.), Mem. SAIt 83 N. 2, 440.

[75] Greco, M. (ed.): 2009, 2010, Le Rencontres de Physique de la Vallée d'Aoste: Results and Perspectives in Particle Physics, Frascati Phys. Ser. Vol. L, Vol. LI.

[76] Greiner, J. et al.: 2009, ApJ 693, 1610.

[77] Gustavino, C.: 2007, in Frontier Objects in Astrophysics and Particle Physics, F. Giovannelli \& G. Mannocchi (eds.), Italian Physical Society, Ed. Compositori, Bologna, Italy, 93, 191.

[78] Gustavino, C.: 2009, in Frontier Objects in Astrophysics and Particle Physics, F. Giovannelli \& G. Mannocchi (eds.), Italian Physical Society, Ed. Compositori, Bologna, Italy, 98, 77.

[79] Gustavino, C.: 2011, in Frontier Objects in Astrophysics and Particle Physics, F. Giovannelli \& G. Mannocchi (eds.), Italian Physical Society, Ed. Compositori, Bologna, Italy, 103, 657.

[80] Hess, V.F.: 1912, Physik Zh. 13, 1084.

[81] van den Heuvel, E.P.J.: 2009, Ap\&SS Library 359, 125.

[82] Hillas, A.M.: 1999, in Frontier Objects in Astrophysics and Particle Physics, F. Giovannelli \& G. Mannocchi (eds.), Italian Physical Society, Editrice Compositori, Bologna, Italy 65, 391.

[83] Hillas, A.M., Johnson, A.P.: 1990, Proc. 21st Intern. Cosmic Ray Conf. (Adelaide) 4, 19.

[84] Hurley, K.: 2008, ChJA\&AS 8, 202.

[85] Izzo, L. et al.: 2010, J. Korean Phys. Soc. 57, No. 3, 551.

[86] Johnstone, Wm.R.: 2005, http://www.johnstonsarchive.net/ relativity/binpulstable.html

[87] Kappes, A., Hinton, J., Stegman, C., Aharonian, F.A.: 2007, ApJ 656, 870.

[88] Kuiper, L.: 2007, Talk presented at the Frascati Workshop on Multifrquency Behaviour of High Energy Cosmic Sources.

[89] Kulikov, G.V., Khristiansen, G.B.: 1958, JETP 35, 63.

[90] LaRosa, T.N., Kassim, N.E., Lazio, T.J.W., Hyman, S.D.: 2000, AJ 119, 207.

[91] Lawrence, M.A., Reid, R.J.O., Watson, A.A.: 1991, J. Phys. G: Nucl. Part. Phys. 17, 733.

[92] Lenain, J.-P.: 2010, in Multifrequency Behaviour of High Energy Cosmic Sources, F. Giovannelli \& L. Sabau-Graziati (eds.), Mem. SAIt 81 N. 1, 362.

[93] Martinez, M.: 2007, Ap\&SS 309, 477.

[94] Mather, J.C. et al.: 1994, ApJ 420, 439.

[95] Mereghetti, S., Stella, L.: 1995, ApJL 442, L17.

[96] Meszaros, P., Rees, M.J.: 1992, ApJ 397, 570.

[97] Mezger, P.G., Duschl, W.J., Zylka, R.: 1996, A\&A Rev. 7, 289.

[98] Mezzetto, M.: 2011, Journal of Physics: Conference Series 335, 012005.

[99] Morselli, A.: 2007, in High Energy Physics ICHEP '06, Y. Sissakian, G. Kozlov \& E. Kolganova (eds.), World Sci. Pub. Co., p. 222.
[100] Müller, D.: 1993, in Frontier Objects in Astrophysics and Particle Physics, F. Giovannelli, G. Mannocchi (eds.), Italian Physical Society, Editrice Compositori, Bologna, Italy 40, 391.

[101] Nagano, M. et al.: 1992, J. Phys. G: Nucl. Part. Phys. 18, 423.

[102] Nagano, M., Watson, A.A.: 2000, Rev. Mod. Phys. 72,689 .

[103] Ouchi, M. et al.: 2010, ApJ 723, 869.

[104] Palanque-Delabrouille, N.: 2003, in Frontier Objects in Astrophysics and Particle Physics, F. Giovannelli \& G. Mannocchi (eds.), Italian Physical Society, Ed. Compositori, Bologna, Italy, 85, 131.

[105] van Paradijs, J.: 1998, in The Many Faces of Neutron Stars, R. Buccheri, J. van Paradijs \& and M.A. Alpar (eds.), Kluwer Academic Publ., Dordrecht, Holland, p. 279.

[106] van Paradijs, J., Taam, R.E., van den Heuvel, E.P.J.: 1995, A\&A 299, L41.

[107] Paredes, J.M., Persic, M.: 2010, in Multifrequency Behaviour of High Energy Cosmic Sources, F. Giovannelli \& L. Sabau-Graziati (eds.), Mem. SAIt 81 N. 1, 204.

[108] Piran, T.: 1999, Phys. Rep. 314, 575.

[109] Piran, T.: 2010, arXiv1005.3311.

[110] Rahoui, F., Chaty, S., Lagage, P.-O, Pantin, E.: 2008, A\&A 484, 801.

[111] Ressel, M.T., Turner, M.S.: 1990, Comm. Astrophys. 14, 323.

[112] Ruffini, R. et al.: 2003, AIP Conf. Proc. 668, 16.

[113] Schroedter, M.: 2005, ApJ 628, 617.

[114] Shibata, M.: 2008, Nuclear Physics B (Proc. Suppl.) $175 \ddot{\jmath} £ ¡ 176,267$.

[115] Spergel, D.N. et al.: 2003, ApJS 148, 175.

[116] Srianand, R., Petitjean, P., Ledoux, C.: 2000, Nature 408, 931.

[117] Stanev, T.: 2001, in Frontier Objects in Astrophysics and Particle Physics, F. Giovannelli \& G. Mannocchi (eds.), Italian Physical Society, Editrice Compositori, Bologna, Italy 73, 397.

[118] Straessner, A. (on behalf of the ATLAS Collaboration): 2011, in Frontier Objects in Astrophysics and Particle Physics, F. Giovannelli \& G. Mannocchi (eds.), Italian Physical Society, Ed. Compositori, Bologna, Italy, 103, 43.

[119] Tanvir, N.R. et al.: 2009, Nature 461, 1254.

[120] Tavani, M. et al.: 2011, Science 331, 736.

[121] Taylor, A.M. et al.: 2008, in High Energy Gamma-Ray Astronomy, AIP Conf. Proc. 1085, 384.

[122] Tozzi, P. et al.: 2003, ApJ 593, 705.

[123] Wang, F.Y., Dai, Z.G.: 2009, MNRAS 400, 10.

[124] Wollack, J.: 2011, NASA Official Web page.

[125] Xu, X.W. (IceCube Collaboration): 2008, N. Phys. B $175-176,401$.

[126] Zatsepin, V.I.: 1995, J. Phys. G: Nucl. Part. Phys. 21, Issue 5, L31. 